ANNALES

POLONICI MATHEMATICI

$83.3(2004)$

\title{
Existence of solutions to generalized von Foerster equations with functional dependence
}

\author{
by HenRYk Leszczyński (Gdańsk) and Piotr Zwierkowski (Toruń)
}

\begin{abstract}
We prove the existence of solutions to a differential-functional system which describes a wide class of multi-component populations dependent on their past time and state densities and on their total size. Using two different types of the Hale operator, we incorporate in this model classical von Foerster-type equations as well as delays (past time dependence) and integrals (e.g. influence of a group of species).
\end{abstract}

Introduction. The problem considered in this paper originates from biology, medicine and chemistry. The independent variables $x_{j}$ and unknown functions $u_{i}$ stand for certain features and densities, respectively. It follows from this natural interpretation that $x_{j} \geq 0$ and $u_{i} \geq 0$.

Some existence, uniqueness and qualitative theory for von Foerster type problems has been established in $[4,5,7-9]$. Other related problems of mathematical biology can be found in [1, 15, 17-19, 21, 22]. Some first-order partial differential functional existence and uniqueness results are published in $[13,14,16]$.

We generalize the previous results in the following ways: (i) including many species and many space variables, (ii) taking into consideration past densities $u_{i}(\cdot)$ and past total sizes of species $\int u_{i}(\cdot, x) d x$, (iii) modifying and weakening some assumptions.

As in $[4,5]$, we study only the case of tangent bicharacteristics at the lateral boundaries, where $x_{j}=0$ for some $j$. Therefore it is convenient to extend the initial data $v_{i}$ to the zone $E_{0}=\left[-\tau_{0}, 0\right] \times \mathbb{R}^{n}\left(\tau_{0} \geq 0\right)$ so as to satisfy the symmetry condition

$$
v_{i}(t, x)=v_{i}(t,|x|) \quad \text { for }(t, x) \in E_{0},|x|:=\left(\left|x_{1}\right|, \ldots,\left|x_{n}\right|\right) \in \mathbb{R}^{n} .
$$

We will look for symmetric, nonnegative solutions $u_{i}$ in the whole zone $E:=[0, a] \times \mathbb{R}^{n}(a>0)$. This simple idea occurs to be very fruitful, since

2000 Mathematics Subject Classification: Primary 35R10; Secondary 35L45, 35A05, $92 \mathrm{~B} 05$.

Key words and phrases: existence, differential functional, von Foerster equation, Hale operator. 
a bounded domain (and unbounded domain with no symmetry, e.g. $\mathbb{R}_{+}^{n}$ ) causes superfluous constraints on any functional model. We expect that these functions shall be continuous in $(t, x)$ and summable in $x$. The process is considered as an evolution

$$
t \mapsto u(t, \cdot) \in L^{1}\left(\mathbb{R}^{n}, \mathbb{R}_{+}^{m}\right) \cap C B\left(\mathbb{R}^{n}, \mathbb{R}_{+}^{m}\right)
$$

(nonnegative, summable and bounded continuous functions).

Let $\tau=\left(\tau_{1}, \ldots, \tau_{n}\right) \in \mathbb{R}_{+}^{n}$, where $\mathbb{R}_{+}:=[0, \infty)$. Define

$$
B=\left[-\tau_{0}, 0\right] \times[-\tau, \tau], \quad \text { where }[-\tau, \tau]=\left[-\tau_{1}, \tau_{1}\right] \times \cdots \times\left[-\tau_{n}, \tau_{n}\right] .
$$

For each function $w$ defined on $\left[-\tau_{0}, a\right]$, we have the Hale functional $w_{t}$ (see $[10]$ ), which is the function defined on $\left[-\tau_{0}, 0\right]$ by

$$
w_{t}(s)=w(t+s) \quad\left(s \in\left[-\tau_{0}, 0\right]\right) .
$$

For each function $u$ defined on $E_{0} \cup E$, we similarly introduce a Hale-type functional $u_{(t, x)}$, defined on $B$ by

$$
u_{(t, x)}(s, y)=u(t+s, x+y) \quad \text { for }(s, y) \in B
$$

(see [13]). Let

$$
\Omega_{0}=E \times C\left(\left[-\tau_{0}, a\right], \mathbb{R}_{+}^{m}\right), \quad \Omega=E \times C\left(B, \mathbb{R}_{+}^{m}\right) \times C\left(\left[-\tau_{0}, a\right], \mathbb{R}_{+}^{m}\right) .
$$

Take $v=\left(v_{1}, \ldots, v_{m}\right): E_{0} \rightarrow \mathbb{R}_{+}^{m}$ and

$$
c_{i j}: \Omega_{0} \rightarrow \mathbb{R}, \lambda_{i}: \Omega \rightarrow \mathbb{R} \quad(i=1, \ldots, m, j=1, \ldots, n) .
$$

We consider the system of differential-functional equations

$$
\frac{\partial u_{i}}{\partial t}+\sum_{j=1}^{n} c_{i j}\left(t, x, z[u]_{t}\right) \frac{\partial u_{i}}{\partial x_{j}}=\lambda_{i}\left(t, x, u_{(t, x)}, z[u]_{t}\right) \quad(i=1, \ldots, m),
$$

where

$$
z[u](t):=\int_{\mathbb{R}^{n}} u(t, y) d y, \quad t \in\left[-\tau_{0}, a\right], u=\left(u_{1}, \ldots, u_{m}\right),
$$

with the initial conditions

$$
u(t, x)=v(t, x), \quad(t, x) \in E_{0}, x=\left(x_{1}, \ldots, x_{n}\right) \in \mathbb{R}^{n} .
$$

We are looking for Carathéodory's solutions to $(1)-(3)$ (see $[4,5])$. The functional dependence includes a possible delayed and integral dependence of the Volterra type. The Hale functional $z[u]_{t}$ takes into consideration the whole population within the time interval $\left[t-\tau_{0}, t\right]$, whereas the Hale-type functional $u_{(t, x)}$ shows the dependence on the density $u=\left(u_{1}, \ldots, u_{m}\right)$ locally in a neighbourhood of $(t, x)$. In particular, our results generalize $[4,5]$. Observe that the functional dependence demands some initial data on a "thick" initial set $E_{0}$, which means that a complicated ecological niche must be observed for some time and (perhaps) in some space in order to determine and predict its further evolution. 
ExAMPLE 0.1. The functional dependence in (1), represented by the Hale operators, generalizes von Foerster equations with delays, deviations and integrals, such as the equation with delays

$$
\begin{aligned}
\frac{\partial u_{i}}{\partial t}+\sum_{j=1}^{n} \bar{c}_{i j}(t, x, & z[u](\beta(t))) \frac{\partial u_{i}}{\partial x_{j}} \\
& =u_{i} \bar{\lambda}_{i}(t, x, u(\bar{\alpha}(t, x)), z[u](\bar{\beta}(t))) \quad(i=1, \ldots, m),
\end{aligned}
$$

where $\bar{\alpha}(t, x)=\left(\bar{\alpha}_{0}(t, x), \ldots, \bar{\alpha}_{n}(t, x)\right), \bar{\alpha}_{0}(t, x) \leq t$ and $\beta(t), \bar{\beta}(t) \leq t$; and the equation with integrals

$$
\begin{aligned}
\frac{\partial u_{i}}{\partial t}+\sum_{j=1}^{n} & \bar{c}_{i j}\left(t, x, \int_{t-\tau_{0}}^{t} z[u](s) d s\right) \frac{\partial u_{i}}{\partial x_{j}} \\
& =u_{i} \bar{\lambda}_{i}\left(t, x, \int_{[x, x+\tau]} u(t, y) d y, \int_{t / 3}^{t} z[u](s) d s\right) \quad(i=1, \ldots, m),
\end{aligned}
$$

where $\bar{c}_{i j}: E \times \mathbb{R}_{+}^{m} \rightarrow \mathbb{R}$ and $\bar{\lambda}_{i}: E \times \mathbb{R}_{+}^{m} \times \mathbb{R}_{+}^{m} \rightarrow \mathbb{R}$.

The paper is organized as follows:

(i) first, we analyse existence and key properties of bicharacteristics;

(ii) we write a solution of problem (1), (3) along bicharacteristics, for a given function $z$, which belongs to a given class under natural assumptions on the data;

(iii) next, considering solutions along those bicharacteristics, we get an integral fixed point equation;

(iv) we state the existence of solutions in a closed subset of functions, generated by a priori estimates.

1. Bicharacteristics. Set $c_{i}=\left(c_{i 1}, \ldots, c_{i n}\right)$. First, for a given function $z \in C\left(\left[-\tau_{0}, a\right], \mathbb{R}_{+}^{m}\right)$, we consider the bicharacteristic equations for problem $(1),(3)$ :

$$
\eta_{i}^{\prime}(s)=c_{i}\left(s, \eta_{i}(s), z_{s}\right), \quad \eta_{i}(t)=x \quad(i=1, \ldots, m) .
$$

Denote by

$$
\eta_{i}=\eta_{i}[z](\cdot ; t, x)=\left(\eta_{i 1}[z](\cdot ; t, x), \ldots, \eta_{i n}[z](\cdot ; t, x)\right)
$$

the $i$ th bicharacteristic curve passing through $(t, x) \in E$, i.e., the solution to problem (4). Next, we consider the equation

$$
\frac{d}{d s} u_{i}\left(s, \eta_{i}[z](s ; t, x)\right)=\lambda_{i}\left(s, \eta_{i}[z](s ; t, x), u_{\left(s, \eta_{i}[z](s ; t, x)\right)}, z_{s}\right)
$$

with the initial condition

$$
u_{i}\left(0, \eta_{i}[z](0 ; t, x)\right)=v_{i}\left(0, \eta_{i}[z](0 ; t, x)\right)
$$


A solution of the above equation is a solution of problem (1), (3) for a given function $z \in C\left(\left[-\tau_{0}, a\right], \mathbb{R}_{+}^{m}\right)$.

Assume that:

(V0) $v_{i} \in C B\left(E_{0}, \mathbb{R}_{+}\right)$for $i=1, \ldots, m$ (nonnegative, bounded and continuous functions).

(V1) $z[v] \in C\left(\left[-\tau_{0}, 0\right], \mathbb{R}_{+}^{m}\right)$, where

$$
z_{i}[v](t)=\int_{\mathbb{R}^{n}} v_{i}(t, x) d x<\infty \quad(i=1, \ldots, m) .
$$

(C0) $\quad c_{i j}: \Omega_{0} \rightarrow \mathbb{R}$ are continuous in $(t, x, q)$ and locally Lipschitz continuous in $x$.

(C1) $\quad \operatorname{tr} \partial_{x} c_{i}(t, x, q) \leq b(t)$ with some $b \in L^{1}\left([0, a], \mathbb{R}_{+}\right)$.

$(\Lambda 0) \quad \lambda_{i}: \Omega \rightarrow \mathbb{R}$ are continuous in $(t, x, w, q)$ and locally Lipschitz continuous in $w$.

$(\Lambda 1) \quad \lambda_{i}(t, x, w, q)=w(0,0) \Lambda_{i}(t, x, w, q)$ for $(t, x) \in E, w \in C\left(B, \mathbb{R}_{+}^{m}\right)$, $q \in C\left(\left[-\tau_{0}, a\right], \mathbb{R}_{+}^{m}\right)$.

REMARK 1.1. Continuity in $t$ in the above assumptions can be weakend to local Lebesgue integrability in $t$, i.e., typical Carathéodory conditions.

$(\Lambda 2) \quad$ There exists $L_{\Lambda} \in L^{1}\left([0, a], \mathbb{R}_{+}\right)$such that

$$
\Lambda_{i}(t, x, w, q) \leq L_{\Lambda}(t)
$$

for $i=1, \ldots, m$ and $(t, x) \in E, w \in C\left(B, \mathbb{R}_{+}^{m}\right), q \in C\left(\left[-\tau_{0}, a\right], \mathbb{R}_{+}^{m}\right)$.

Define

$$
W_{i}(t, x, w, q)=\Lambda_{i}(t, x, w, q)+\operatorname{tr} \partial_{x} c_{i}(t, x, q)
$$

for $i=1, \ldots, m$ and $(t, x) \in E, w \in C\left(B, \mathbb{R}_{+}^{m}\right), q \in C\left(\left[-\tau_{0}, a\right], \mathbb{R}_{+}^{m}\right)$.

(W1) There exists $L_{W} \in L^{1}\left([0, a], \mathbb{R}_{+}\right)$such that

$$
W_{i}(t, x, w, q) \leq L_{W}(t)
$$

for $i=1, \ldots, m$ and $(t, x) \in E, w \in C\left(B, \mathbb{R}_{+}^{m}\right), q \in C\left(\left[-\tau_{0}, a\right], \mathbb{R}_{+}^{m}\right)$.

(W2) The mapping

$$
(t, x, w, q) \mapsto \int_{0}^{t} W_{i}(s, x, w, q) d s
$$

transforms bounded subsets of $E \times C\left(B, \mathbb{R}_{+}^{m}\right) \times C\left(\left[-\tau_{0}, a\right], \mathbb{R}_{+}^{m}\right)$ to bounded subsets of $\mathbb{R}$.

(W3) The mapping

$$
(t, x, w, q) \mapsto \int_{0}^{t} W_{i}(s, x, w, q) d s
$$

transforms bounded subsets of $E \times C\left(B, \mathbb{R}_{+}^{m}\right) \times C\left(\left[-\tau_{0}, a\right], \mathbb{R}_{+}^{m}\right)$ to sets of uniformly continuous functions with respect to $(t, x)$. 
REMARK 1.2. Assumption (W3) is valid if the functional arguments become classical. This assumption is also satisfied if the functions $W_{i}, i=$ $1, \ldots, m$, are uniformly continuous with respect to $x, w$ and $q$.

REMARK 1.3. Note that if for a given function $z \in C\left(\left[-\tau_{0}, a\right], \mathbb{R}_{+}^{m}\right)$, the right-hand side in (4) satisfies the local Lipschitz condition in $x$, then there exists a unique solution to (4). This is also valid for (5) if the function $\lambda_{i}$ is locally Lipschitz in $w$ (see $[3,11,12])$.

LEMMA 1.1. If Assumptions (V0), (V1), (C0), (C1) are satisfied, then

$$
\widetilde{v}_{i}(t):=\int_{\mathbb{R}^{n}} v_{i}\left(0, \eta_{i}(0 ; t, x)\right) d x \leq z_{i}[v](0) \exp \left(\int_{0}^{t} b(s) d s\right)<\infty
$$

where $\eta_{i}(s ; t, x)=\eta_{i}[z](s ; t, x)$.

Proof. Define

$$
\begin{aligned}
G_{i}(s ; t, x) & =\left[\partial_{x_{l}} \eta_{i j}[z](s ; t, x)\right]_{j, l=1, \ldots, n}, \\
Q_{i}(s ; t, x) & =\left(s, \eta_{i}[z](s ; t, x), z_{s}\right), \\
C_{i}(s ; t, x) & =\left[\partial_{x_{l}} c_{i j}\left(Q_{i}(s ; t, x)\right)\right]_{l, j=1, \ldots, n} .
\end{aligned}
$$

By the Liouville theorem, it follows from (4) that

$$
\operatorname{det} G_{i}(s ; t, x)=\exp \left(-\int_{s}^{t} \operatorname{tr} C_{i}(\sigma ; t, x) d \sigma\right)
$$

Applying this to the integral $\widetilde{v}_{i}(t)$ together with $(\mathrm{C} 1)$, we get

$$
\widetilde{v}_{i}(t) \leq \int_{\mathbb{R}^{n}}\left(v_{i}\left(0, \eta_{i}(0 ; t, x)\right) / \operatorname{det} G_{i}(0 ; t, x)\right) d x \leq z_{i}[v](0) \exp \left(\int_{0}^{t} b(s) d s\right) \text {. }
$$

LEMMA 1.2. If $u$ is a solution of (5) and Assumptions (V0), ( $\Lambda 1),(\Lambda 2)$ are satisfied, then

$$
0 \leq u_{i}(t, x) \leq\|v(0, \cdot)\|_{\infty} \exp \left(\int_{0}^{t} L_{\Lambda}(s) d s\right)
$$

Proof. Due to $(\Lambda 1)$, the solution of problem (5) is given by the formula

$$
u_{i}(t, x)=v_{i}\left(0, \eta_{i}(0)\right) \exp \left(\int_{0}^{t} \Lambda_{i}\left(s, \eta_{i}(s), u_{\left(s, \eta_{i}(s)\right)}, z_{s}\right) d s\right),
$$

where $\left.\eta_{i}(s)=\eta_{i}(s ; t, x)\right)$. Since $v_{i}(0, x) \geq 0$, it follows that $u_{i}(t, x) \geq 0$ for $i=1, \ldots, m$ and $(t, x) \in E$.

2. The fixed point equation. In order to prove the existence for problem (1)-(3) we apply Schauder's fixed point theorem. We define a convex, 
closed set and an integral operator for which we show the existence of a fixed point. This will be preceded by three auxiliary lemmas. Define

$$
\mathcal{Z}=\left\{z \in C\left(\left[-\tau_{0}, a\right], \mathbb{R}_{+}^{m}\right): 0 \leq z(t) \leq Z(t) \text { for all } t\right\},
$$

where

$$
Z(t)= \begin{cases}\max _{-\tau_{0} \leq s \leq 0}\|v(s, \cdot)\|_{L^{1}} & \text { for } t \in\left[-\tau_{0}, 0\right], \\ Z(0) \exp \left(\int_{0}^{t} L_{W}(s) d s\right) & \text { for } t \in[0, a] .\end{cases}
$$

Consider the operator $\mathcal{T}: \mathcal{Z} \rightarrow \mathcal{Z}$ given by

$$
\begin{array}{ll}
\mathcal{T}_{i}[z](t)=\int_{\mathbb{R}^{n}} v_{i}(t, x) d x & \text { for } t \leq 0, \\
\mathcal{T}_{i}[z](t)=\int_{\mathbb{R}^{n}} u_{i}[z](t, x) d x & \text { for } t \geq 0,
\end{array}
$$

where $u \in C^{1}\left(B, \mathbb{R}_{+}^{m}\right)$ is the solution of (5).

By Lemma 1.2 we write (10) as

$$
\mathcal{T}_{i}[z](t)=\int_{\mathbb{R}^{n}} v_{i}\left(0, \eta_{i}(0)\right) \exp \left(\int_{0}^{t} \Lambda_{i}\left(s, \eta_{i}(s), u_{\left(s, \eta_{i}(s)\right)}, z_{s}\right) d s\right) d x
$$

for $t \geq 0$, where $\eta_{i}(s)=\eta_{i}[z](s ; t, x)$. Notice that the bicharacteristics have the following group property:

$$
y=\eta_{i}[z](0 ; t, x) \Leftrightarrow \eta_{i}[z](s ; t, x)=\eta_{i}[z](s ; 0, y), \quad i=1, \ldots, m,
$$

that is, any $i$ th bicharacteristic curve passing through $(0, y)$ and $(t, x)$ has the same value at all $s \in[0, a]$.

The change of variables $y=\eta_{i}[z](0 ; t, x)$ and the Liouville theorem show that the Jacobian $J=\operatorname{det}\left[\partial c_{i} / \partial x\right]$ is given by the formula

$$
J(0 ; t, x)=\exp \left(-\int_{0}^{t} \operatorname{tr} \partial_{x} c_{i}\left(s, \eta_{i}[z](s ; 0, y), z_{s}\right) d s\right) .
$$

Hence we can write (11) in the form

$$
\mathcal{T}_{i}[z](t)=\int_{\mathbb{R}^{n}} v_{i}(0, y) \exp \left(\int_{0}^{t} W_{i}\left(s, \eta_{i}(s), u_{\left(s, \eta_{i}(s)\right)}, z_{s}\right)\right) d s d y,
$$

where $\eta_{i}(s)=\eta_{i}[z](s ; 0, y)$.

Lemma 2.1. If Assumptions (V1), (C1), ( $\Lambda 1)$ and (W1) are satisfied, then

$$
0 \leq \mathcal{T}_{i}[z](t) \leq Z(t)<\infty \quad \text { for } i=1, \ldots, m, t \in[0, a] .
$$

Proof. This follows directly from (12) and the assumptions. 
Lemma 2.2. If Assumptions (V0), (V1), (C0), (C1), ( 11$)$, (W1) and (W2) are satisfied, then the range $\mathcal{T}(\mathcal{Z})$ is equicontinuous.

Proof. Let $z \in \mathcal{Z}$ and $0 \leq t \leq \bar{t} \leq a$. Set $\eta_{i}(s)=\eta_{i}[z](s ; 0, y)$. If we apply (12), we can write

$$
\begin{aligned}
z_{i}(\bar{t})-z_{i}(t)=\int_{\mathbb{R}^{n}} v_{i}(0, y)\left\{\exp \left(\int_{0}^{\bar{t}} W_{i}\left(s, \eta_{i}(s), u_{\left(s, \eta_{i}(s)\right)}, z_{s}\right) d s\right)\right. \\
\left.\quad-\exp \left(\int_{0}^{t} W_{i}\left(s, \eta_{i}(s), u_{\left(s, \eta_{i}(s)\right)}, z_{s}\right) d s\right)\right\} d y
\end{aligned}
$$

for $t, \bar{t} \in[0, a]$. From Assumptions (V1) and (W1) we obtain

$$
\begin{aligned}
-\int_{\mathbb{R}^{n}} v_{i}(0, y) \exp \left(\int_{0}^{\bar{t}} L_{W}(s) d s\right) d y & \leq z_{i}(\bar{t})-z_{i}(t) \\
& \leq \int_{\mathbb{R}^{n}} v_{i}(0, y) \exp \left(\int_{0}^{t} L_{W}(s) d s\right) d y .
\end{aligned}
$$

Using the mean value theorem, we get the equality

$$
\begin{aligned}
\exp \left(\int_{0}^{\bar{t}} W_{i}\left(s, \eta_{i}(s), u_{\left(s, \eta_{i}(s)\right)}, z_{s}\right) d s\right) & -\exp \left(\int_{0}^{t} W_{i}\left(s, \eta_{i}(s), u_{\left(s, \eta_{i}(s)\right)}, z_{s}\right) d s\right) \\
= & \int_{0}^{1}\left(\operatorname { e x p } \left(\zeta \int_{0}^{t} W_{i}\left(s, \eta_{i}(s), u_{\left(s, \eta_{i}(s)\right)}, z_{s}\right) d s\right.\right. \\
& \left.\left.+(1-\zeta) \int_{0}^{\bar{t}} W_{i}\left(s, \eta_{i}(s), u_{\left(s, \eta_{i}(s)\right)}, z_{s}\right) d s\right)\right) d \zeta \\
& \times \int_{t}^{\bar{t}} W_{i}\left(s, \eta_{i}(s), u_{\left(s, \eta_{i}(s)\right)}, z_{s}\right) d s
\end{aligned}
$$

Taking into consideration the last two relations, we obtain the estimate

$$
\begin{aligned}
&\left|z_{i}(\bar{t})-z_{i}(t)\right| \leq \int_{\mathbb{R}^{n}} v_{i}(0, y) d y \min \left\{\exp \int_{0}^{\bar{t}} L_{W}(s) d s\right. \\
&\left.\exp \left(\int_{0}^{\bar{t}} L_{W}(s) d s\right) \int_{t}^{\bar{t}}\left|W_{i}\left(s, \eta_{i}(s), u_{\left(s, \eta_{i}(s)\right)}, z_{s}\right)\right| d s\right\} .
\end{aligned}
$$

The functions $\left|W_{i}(t, x, w, q)\right|$ are uniformly bounded on the set $E \times C\left(B, \mathbb{R}_{+}^{m}\right)$ $\times C\left(\left[-\tau_{0}, a\right], \mathbb{R}_{+}^{m}\right)$ and depend only on functions in $\mathcal{Z}$. Hence, by Lemma 1.1 and by Lebesgue's dominated convergence theorem $[6], \mathcal{T}[\mathcal{Z}]$ is equicontinuous.

Now we prove the continuity of $\mathcal{T}$ on $\mathcal{Z}$. 
Lemma 2.3. If Assumptions (V0), (V1), (C0), (C1), ( $\Lambda 0),(\Lambda 1),(\mathrm{W} 1)$ and (W3) are satisfied, then the operator $\mathcal{T}$ is continuous on the set $\mathcal{Z}$.

Proof. Take $z, \bar{z} \in \mathcal{Z}$. We shall show that $\left\|\mathcal{T}_{i} z-\mathcal{T}_{i} \bar{z}\right\|_{\infty}$ tends to 0 as $\|z-\bar{z}\|_{\infty} \rightarrow 0$. For each $t \in[0, a]$ we have

$$
\begin{aligned}
& \mathcal{T}_{i}[z](t)-\mathcal{T}_{i}[\bar{z}](t) \\
& =\int_{\mathbb{R}^{n}} v_{i}\left(0, \eta_{i}[z](0 ; t, x)\right) \exp \left(\int_{0}^{t} \Lambda_{i}\left(s, \eta_{i}[z](s ; t, x), u_{\left(s, \eta_{i}[z](s ; t, x)\right)}, z_{s}\right) d s\right) d x \\
& \quad-\int_{\mathbb{R}^{n}} v_{i}\left(0, \eta_{i}[\bar{z}](0 ; t, x)\right) \exp \left(\int_{0}^{t} \Lambda_{i}\left(s, \eta_{i}[\bar{z}](s ; t, x), u_{\left(s, \eta_{i}[\bar{z}](s ; t, x)\right)}, \bar{z}_{s}\right) d s\right) d x .
\end{aligned}
$$

By the change of variables $y=\eta_{i}[z](0 ; t, x)$ and $y=\eta_{i}[\bar{z}](0 ; t, x)$, respectively, we obtain

$$
\begin{aligned}
\mathcal{T}_{i}[z](t)- & \mathcal{T}_{i}[\bar{z}](t) \\
= & \int_{\mathbb{R}^{n}} v_{i}(0, y)\left[\exp \left(\int_{0}^{t} W_{i}\left(s, \eta_{i}[z](s ; 0, y), u_{\left(s, \eta_{i}[z](s ; 0, y)\right)}, z_{s}\right) d s\right)\right. \\
& \left.\quad-\exp \left(\int_{0}^{t} W_{i}\left(s, \eta_{i}[\bar{z}](s ; 0, y), u_{\left(s, \eta_{i}[\bar{z}](s ; 0, y)\right)}, \bar{z}_{s}\right) d s\right)\right] d y .
\end{aligned}
$$

As in the proof of Lemma 2.2, we have

$$
\left|\mathcal{T}_{i}[z](t)-\mathcal{T}_{i}[\bar{z}](t)\right| \leq \int_{\mathbb{R}^{n}} v_{i}(0, y) \exp \left(\int_{0}^{t} L_{W}(s) d s\right) d y .
$$

The mean value theorem yields the estimate

$$
\begin{aligned}
\left|\mathcal{T}_{i}[z](t)-\mathcal{T}_{i}[\bar{z}](t)\right| \leq & \int_{\mathbb{R}^{n}} v_{i}(0, y) \exp \left(\int_{0}^{t} L_{W}(s) d s\right) \\
& \times \min \left\{1, \int_{0}^{t} \mid W_{i}\left(s, \eta_{i}[z](s ; 0, y), u_{\left(s, \eta_{i}[z](s ; 0, y)\right)}, z_{s}\right)\right. \\
& \left.-W_{i}\left(s, \eta_{i}[\bar{z}](s ; 0, y), u_{\left(s, \eta_{i}[\bar{z}](s ; 0, y)\right)}, \bar{z}_{s}\right) \mid d s\right\} d y .
\end{aligned}
$$

By the continuity Assumptions $(\mathrm{C} 0),(\Lambda 0)$ and Lebesgue's dominated convergence theorem, the difference

$$
\left|W_{i}\left(t, \eta_{i}[z](t ; 0, y), u_{\left(t, \eta_{i}[z](t ; 0, y)\right)}, z_{t}\right)-W_{i}\left(t, \eta_{i}[\bar{z}](t ; 0, y), u_{\left(t, \eta_{i}[\bar{z}](t ; 0, y)\right)}, \bar{z}_{t}\right)\right|
$$

tends to zero, provided $\|z-\bar{z}\|_{\infty} \rightarrow 0$ for $(t, y) \in E$. Hence

$$
\left|\mathcal{T}_{i}[z](t)-\mathcal{T}_{i}[\bar{z}](t)\right| \rightarrow 0 \quad \text { for } i=1, \ldots, m, t \in[0, a] .
$$

Now we show that the operator (10) has a fixed point. 
THEOREM 2.1. If the assumptions of Lemmas 2.1-2.3 are satisfied then the operator $\mathcal{T}$ has a fixed point.

Proof. Notice that the set $\mathcal{Z}$ is nonempty, closed, bounded and convex. Lemmas 2.1 and 2.3 show that $\mathcal{T}$ is a continuous operator that maps $\mathcal{Z}$ to $\mathcal{Z}$. By Lemma 2.2 and the Ascoli-Arzelà theorem (see [2]) the range $\mathcal{T}(\mathcal{Z}$ ) is relatively compact. Hence, by Schauder's theorem (see [23]), $\mathcal{T}$ has a fixed point.

REMARK 2.1. By the properties of the bicharacteristics and from Theorem 2.1, it follows that problem (1)-(3) has at least one solution.

We give two additional assumptions and formulate a uniqueness statement (without proof).

(C2) The functions $c_{i j}: \Omega_{0} \rightarrow \mathbb{R}$ are Lipschitz continuous in $x, q$.

(W4) The functions $W_{i}: \Omega \rightarrow \mathbb{R}$ are Lipschitz continuous in $x, w, q$.

REMARK 2.2. Assumption (W4) is satisfied if the functions $\Lambda_{i}$ are Lipschitz continuous in $x, w, q$ and the functions $\operatorname{tr} \partial_{x} c_{i}$ are Lipschitz continuous in $x, q$.

Proposition 2.1. If Assumptions (V0), (V1), (C2), ( $\Lambda 1)$, (W1) and (W4) are satisfied, then problem (1)-(3) admits at most one solution.

From Remark 2.1 and Proposition 2.1 we obtain the following

Corollary 2.1. If Assumptions (V0), (V1), (C0)-(C2), ( $\Lambda 0)-(\Lambda 2)$ and (W1)-(W4) are satisfied, then there exists a unique solution of problem (1) $-(3)$.

Acknowledgements. The research was supported by the Gdańsk University grant BW 5100-5-0084-3.

\section{References}

[1] F. Brauer and C. Castillo-Chávez, Mathematical Models in Population Biology and Epidemiology, Springer, New York, 2001.

[2] A. L. Brown and A. Page, Elements of Functional Analysis, Van Nostrand Reinhold, London, 1970.

[3] R. Courant, Methods of Mathematical Physics, Vol. II, Partial Differential Equations, Interscience, New York, 1992.

[4] A. L. Dawidowicz, Existence and uniqueness of solution of generalized von Foerster integro-differential equation with multidimensional space of characteristics of maturity, Bull. Polish Acad. Sci. Math. 38 (1990), 1-12.

[5] A. L. Dawidowicz and K. Łoskot, Existence and uniqueness of solution of some integro-differential equation, Ann. Polon. Math. 47 (1986), 79-87.

[6] J. L. Doob, Measure Theory, Springer, New York, 1994.

[7] H. von Foerster, Some remarks on changing populations, in: The Kinetics of Cellular Proliferation, Grune and Stratton, New York, 1959, 382-407. 
[8] M. E. Gurtin, A system of equations for age-dependent population diffusion, J. Theor. Biol. 40 (1973), 389-392.

[9] M. E. Gurtin and R. McCamy, Non-linear age-dependent population dynamics, Arch. Rat. Mech. Anal. 54 (1974), 281-300.

[10] J. K. Hale and S. V. Lunel, Introduction to Functional Differential Equations, Appl. Math. Sci. 99, Springer, New York, 1993.

[11] P. Hartman, Ordinary Differential Equations, Wiley, New York, 1964.

[12] E. Kamke, Differentialgleichungen II, Akad. Verlagsgesellschaft, Leipzig, 1965.

[13] Z. Kamont, Hyperbolic Functional Differential Inequalities and Applications, Kluwer, 1999.

[14] Z. Kamont and H. Leszczyński, Uniqueness result for the Carathéodory solutions to the Cauchy problem for first-order partial differential-functional equations, Z. Anal. Anwend. 13 (1994), 477-492.

[15] N. Keyfitz, Introduction to the Mathematics of Population, Addison-Wesley, Reading, 1968.

[16] H. Leszczyński, On CC-solutions to the initial-boundary-value problem for systems of non-linear first-order partial differential-functional equations, Rend. Mat. 15 (1995), 173-209.

[17] A. J. Lotka, Elements of Physical Biology, Wiliams and Wilkins, Baltimore, 1925; republished as Elements of Mathematical Biology, Dover, New York, 1956.

[18] A. M. Nakhushev, Equations of Mathematical Biology, Vysshaya Shkola, Moscow, 1995 (in Russian).

[19] J. M. Smith, Mathematical Ideas in Biology, Cambridge Univ. Press, 1968.

[20] J. Szarski, Differential Inequalities, PWN, Warszawa, 1965.

[21] P. F. Verhulst, Recherches mathématiques sur la loi d'accroissement de la population, Mém. Acad. Roy. Bruxelles 18.

[22] —, Recherches mathématiques sur la loi d'accroissement de la population, Mém. Acad. Roy. Bruxelles 20.

[23] K. Yosida, Functional Analysis, Springer, Berlin, 1995.

Institute of Mathematics

University of Gdańsk

Wita Stwosza 57

80-952 Gdańsk, Poland

E-mail: hleszcz@math.univ.gda.pl
Faculty of Mathematics and Computer Science Nicolaus Copernicus University Chopina 12/14 87-100 Toruń, Poland E-mail: zwierkow@mat.uni.torun.pl 